\title{
OMEGADAY Softgels: The Superfood for Healthy Heart
}

Govind Shukla, Aishwarya Pothuganti, Sandeep kunche, Anusha kandala,

Arun Kumar Junjipelly \& C. J. Sampath Kumar

Pugos Nutrition Research Centre, Hyderabad. A Unit of PUGOS Products Pvt. Ltd.,

42, $2^{\text {nd }}$ Floor, Leelavathi Mansion, $6^{\text {th }}$ Cross, Margosa Main Road, Malleshwaram, Bangalore-56003, Karnataka, India.

DOI: http://doi.org/10.46382/MJBAS.2021.5207

Copyright: (02021 Govind Shukla et al. This is an open access article distributed under the terms of the Creative Commons Attribution License, which permits unrestricted use, distribution, and reproduction in any medium, provided the original author and source are credited.

ABSTRACT

Heart disease, also known as cardiovascular disease, refers to diseases of the blood vessels and heart. It is number one cause of death in the world, and in $2020,11 \%$ of all deaths were as a result of heart disease. Although there is not one single cause, an unhealthy diet can be one of the contributing risk factors for heart disease. Paying attention to what we eat and consuming a variety of healthy foods from the five food groups is one of the most important preventative measures we can take to make our heart healthy. The present article reviews the role of Omegaday Softgels: The superfood for healthy heart developed by R\& D cell of Pugos Nutrition Research Centre, Hyderabad, as an important preventive measure to make our heart healthy.

\section{Introduction}

Heart disease results from the narrowing of the arteries that supply the heart with blood through a process known as atherosclerosis. Fatty deposits (or plaque) gradually build up on the inside of the artery walls, narrowing the space in which blood can flow to heart. Atherosclerosis can start when we are young, so by the time we reach middle age, it can be quite advanced. Plaque build-up can be considered as stable or unstable. If there is too much build-up of stable plaque, it narrows the arteries, causing pain and discomfort due to not enough blood reaching the heart - this is called angina and it needs to be treated. Unstable plaque is inflamed and has a thin cap which is prone to developing a crack, allowing the blood to come in contact with the fatty contents of the plaque. The blood will clot to try to seal the gap but in doing so, the blood clot blocks the artery. This prevents the flow of blood to the heart, cuts off its oxygen supply and damages or kills the heart cells. This is a heart attack.

\section{Risk Factors for Heart Disease}

\begin{tabular}{|l|l|}
\hline Risk factors that can't be changed & Risk factors within your control \\
\hline Age & Smoking status \\
Gender & Diet \\
Ethnicity & Cholesterol levels \\
Family history of heart disease & Blood pressure \\
& Body weight \\
& Diabetes management \\
& Physical activity levels \\
& Depression and social isolation \\
\hline
\end{tabular}


There are many factors that can increase our risk of heart disease. Although some of these cannot be changed, the good news is that there are plenty of risk factors within your control. For example, by being physically active, ensuring to have good social support and not smoking, our risk of heart disease is reduced.

Some risk factors are connected. For example, cholesterol levels and blood pressure can be affected by diet, as can our body weight and management of diabetes.

Therefore, one of the best things we can do to reduce our risk of heart disease is to have a healthy diet and maintain a healthy weight.

\section{Dietary Fats and Cholesterol Levels}

Cholesterol is a fat crucial to many metabolic functions and is an essential part of all the body's cell membranes. It is made by the body from the food we eat and is produced in the liver.

Blood lipids (fats) that contain cholesterol include low-density lipoprotein (LDL) and high-density lipoprotein (HDL). LDL ('bad') cholesterol can lead to plaque forming in the arteries while HDL ('good') cholesterol helps to remove cholesterol from the body and makes it harder for plaque to form in the arteries.

Saturated fats (also known as 'bad fats') tend to increase LDL ('bad') cholesterol in the blood. Common sources of saturated fats include - animal products (butter, coconut oil, meat fat including lard and dripping, beef, lamb, chicken skin and palm oil), and processed foods like pastries and biscuits.

\section{Full fat or reduced fat dairy?}

Although full fat dairy foods (such as milk, cheese and yoghurt) contain saturated fat, it appears this type of fat has a neutral relationship with heart health.

The Heart Foundation recommends unflavoured milk, yoghurt and cheese can be consumed by the general population but for people who need to lower LDL cholesterol, reduced fat versions should be consumed instead.

Eggs: It was once thought cholesterol naturally found in eggs was bad for heart health. However, research suggests eggs have a neutral relationship with heart health - they neither increase nor decrease the risk of heart disease for the general population.

\section{Trans fats}

Like saturated fats, trans fats tend to increase LDL (bad) cholesterol in the blood but they also tend to reduce HDL (good) cholesterol levels. So, they are more damaging to our health and can increase our risk of cardiovascular diseases (such as heart disease and stroke). Trans fatty acids form when monounsaturated or polyunsaturated vegetable oils are 'hydrogenated' and hardened to form margarines, oils for deep frying and shortening for baked products.

These harder vegetable fats and shortenings are used by the food industry in processed foods (such as cakes and biscuits and deep-fried takeaway meals). Some trans fatty acids also occur naturally in some meats, butter and dairy products. 


\section{Monounsaturated and polyunsaturated fats}

Reduce your risk of heart disease, by replacing energy intake from saturated and trans ('bad fats') in your diet with unsaturated ('good fats').

Substitute butter, coconut and palm oil, lard, dripping and copha) with oils made from seeds or plants (such as olive, avocado, sunflower, canola, safflower, peanut, soybean and sesame).

Other sources of unsaturated fats include unsalted nuts, seeds (including chia, tahini and linseed) and avocado.

\section{Blood pressure and salt (sodium)}

A diet high in salt is linked to hypertension (high blood pressure), which can increase your risk of heart disease and stroke. Most of us consume more than ten times the amount of salt we need to meet our sodium requirements (salt contains sodium and chloride).

Most of the sodium in our diet is not from added salt at the table, but from packaged and processed foods.

Even sweet foods and those that don't taste 'salty' can have much more sodium than you'd expect!

A simple way to cut down on the amount of sodium in your diet is to reduce the amount of processed foods, limit fast food and use herbs and spices for flavour.

\section{We can reduce our heart disease risk with healthy eating}

Eating a variety of foods is beneficial to our health and can help reduce our risk of disease (including heart disease).

Try to eat a wide variety of foods from each of the five food groups, in the amounts recommended. Not only does this help you maintain a healthy and interesting diet, but it provides essential nutrients to the body.

\section{The American Heart Foundation recommends:}

(1) Plenty of vegetables, fruits and whole grains.

(2) A variety of healthy protein sources (especially fish and seafood), legumes (such as beans and lentils), nuts and seeds. Smaller amounts of eggs and lean poultry can also be included in a heart healthy diet. If choosing red meat, make sure it is lean and limit to 1-3 times a week.

(3) Unflavoured milk, yoghurt and cheese. Those with high blood cholesterol should choose reduced fat varieties.

(4) Healthy fat choices - nuts, seeds, avocados, olives and their oils for cooking.

(5) Herbs and spices to flavour foods, instead of adding salt.

Also, be mindful on how much you are eating and whether you are filling up on unhealthy foods. Portion sizes have increased over time and many of us are eating more than we need which can lead to obesity and increase our risk of cardiovascular disease.

Ideally, a healthy plate would include servings of $-1 / 4$ protein, $1 / 4$ carbohydrates and $1 / 2$ vegetables. 
Remember, serving sizes can vary depending on your age, gender and specific nutrition needs.

\section{Foods important for heart health}

Although there is no one 'magic' food to lower our risk of developing heart disease, there is some evidence that some foods are important for heart health. These include:

- Oily fish - such as mackerel, sardines, tuna and salmon which contain omega-3 fatty acids. This type of fat has been shown to decrease triglycerides (a type of fat) and increase HDL-cholesterol levels, improve blood vessel elasticity and thin the blood, making it less likely to clot and block blood flow.

- Some vegetables oils - such as corn, soy and safflower (which contain omega-6 fatty acids), and those containing omega-3 fatty acids (such as canola and olive oil). All of these can help to lower LDL cholesterol when used instead of saturated fats such as butter.

- Fruit and vegetables - fibre, potassium and other micronutrients (such as antioxidants) in fruit and vegetables offer protection against heart disease. They are also an important source of folate - which helps lower the blood levels of the amino acid homocysteine, which appears to be linked to an increased risk of heart disease.

- Wholegrain - a diet high in fibre from wholegrain cereals is linked to reduced LDL cholesterol and lowered heart disease risk. Foods with high levels of soluble fibre (for example, oats, legumes and barley) are great for lowering total cholesterol levels.

- Unrefined carbohydrate sources with a low glycaemic load - such as wholegrain breads and cereals, legumes, certain types of rice and pasta, and most fruits and vegetables also help to lower blood triglycerides and glucose (sugar) levels, help manage diabetes and reduce heart disease risk. 
- Legumes, nuts and seeds - are good sources of plant proteins, fibre, healthy fats and micronutrients to help lower your cardiovascular risk.

- Tea - some research suggests antioxidants in tea can help prevent the build-up of fatty deposits in the arteries. They may also act as an anti-blood clotting agent and improve blood vessel dilation to allow increased blood flow.

- Foods containing vitamin $\mathbf{E}$ - some studies indicate that vitamin $\mathrm{E}$ acts as an antioxidant, helping to protect against LDL cholesterol.

- Sources of vitamin $\mathbf{E}$ include - avocados, dark green vegetables, vegetable oils and wholegrain products. Eat foods containing vitamin $\mathrm{E}$ rather than supplements, which have not been shown to have the same protective effects.

- Garlic - a compound in fresh garlic (called allicin) has been found to lower total and LDL cholesterol in the blood, thereby reducing the risk of heart disease.

- Foods enriched with plant sterols - a daily intake of 2-3g of phytosterols/stanols lowers LDL cholesterol levels by approximately $10 \%$ in healthy people, those with high cholesterol or diabetes. This equates to 2-3 serves of phytosterol-enriched foods like margarine spreads, yoghurts, milk and breakfast cereals.

\section{How to Reduce Our Risk of Heart Disease with Healthy Eating?}

Try these steps to reduce your risk of developing heart disease:

- Limit fried fast food and processed foods.

- Replace energy from saturated fats (such as butter, coconut oil and cream) with healthy unsaturated fats from seeds and plants (such as extra virgin olive oil, avocado, sunflower, canola, safflower, peanut, soybean and sesame) and foods such as nuts, seeds, avocado, olives and soy.

- Increase the amount and variety of plant foods - eat more vegetables, fruits and wholegrain cereals.

- Reduce intake of refined sources of carbohydrates with higher glycaemic indices (including foods with added sugars).

- Limit unprocessed red meats (such as beef, veal, mutton, lamb, pork, kangaroo, rabbit, and other game meats) to a maximum of $350 \mathrm{~g}$ (cooked weight) per week and avoid processed meat (such as sausages, ham, salami and prosciutto).

- Trim all visible fat from meat and remove skin from poultry.

- Eat legumes regularly - like baked beans (reduced salt), soybeans, lentils and tofu.

- Snack on a handful of raw, unsalted nuts on most days of the week (especially walnuts and almonds).

- Eat oily fish at least once per week. 
- Reduce your salt intake - avoid packaged and processed foods, limit fast foods and salty foods. Replace salt at the table and in cooking, with herbs and spices for flavour.

- Check the sodium content of foods and choose the lowest sodium products.

- If you have elevated cholesterol levels, switch to low-fat or non-fat dairy products and have no more than 7 eggs per week.

- If you drink alcohol, have no more than two standard drinks on any one day. A high alcohol intake increases blood pressure and can increase triglycerides in the blood.

\section{Pathophysiology of Heart Failure}

The main pathophysiology of heart failure is a reduction in the efficiency of the heart muscle, through damage or overloading.

As such, it can be caused by a wide number of conditions, including myocardial infarction (in which the heart muscle is starved of oxygen and dies), hypertension (which increases the force of contraction needed to pump blood) and amyloidosis (in which misfolded proteins are deposited in the heart muscle, causing it to stiffen). Over time these increases in workload will produce changes to the heart itself.

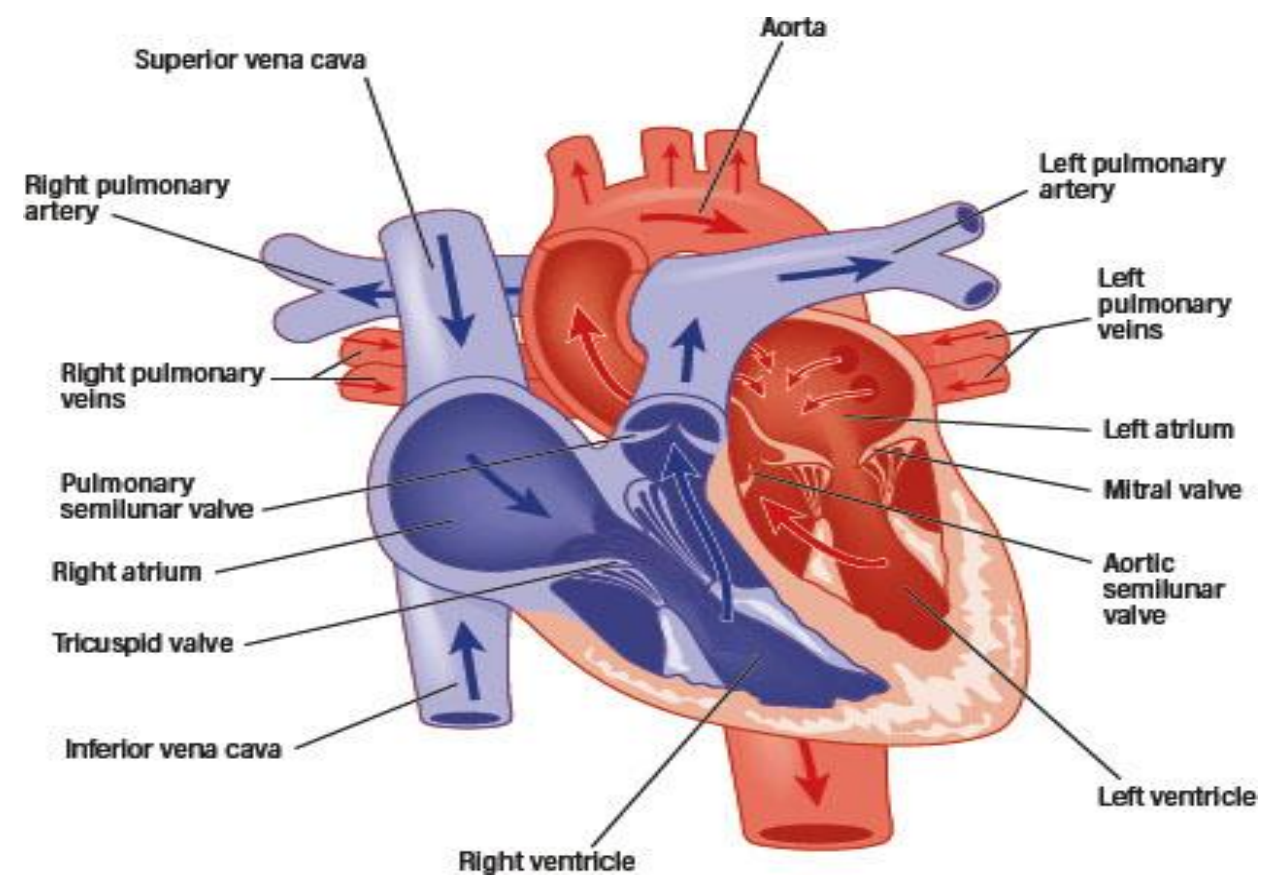

Fig.1.

The heart of a person with heart failure may have a reduced force of contraction due to overloading of the ventricle. In a healthy heart, increased filling of the ventricle results in increased contraction force (by the Frank-Starling law of the heart) and thus a rise in cardiac output.

In heart failure, this mechanism fails, as the ventricle is loaded with blood to the point where heart muscle contraction becomes less efficient. This is due to reduced ability to cross-link actin and myosin filaments in over-stretched heart muscle. ${ }^{[1]}$ 


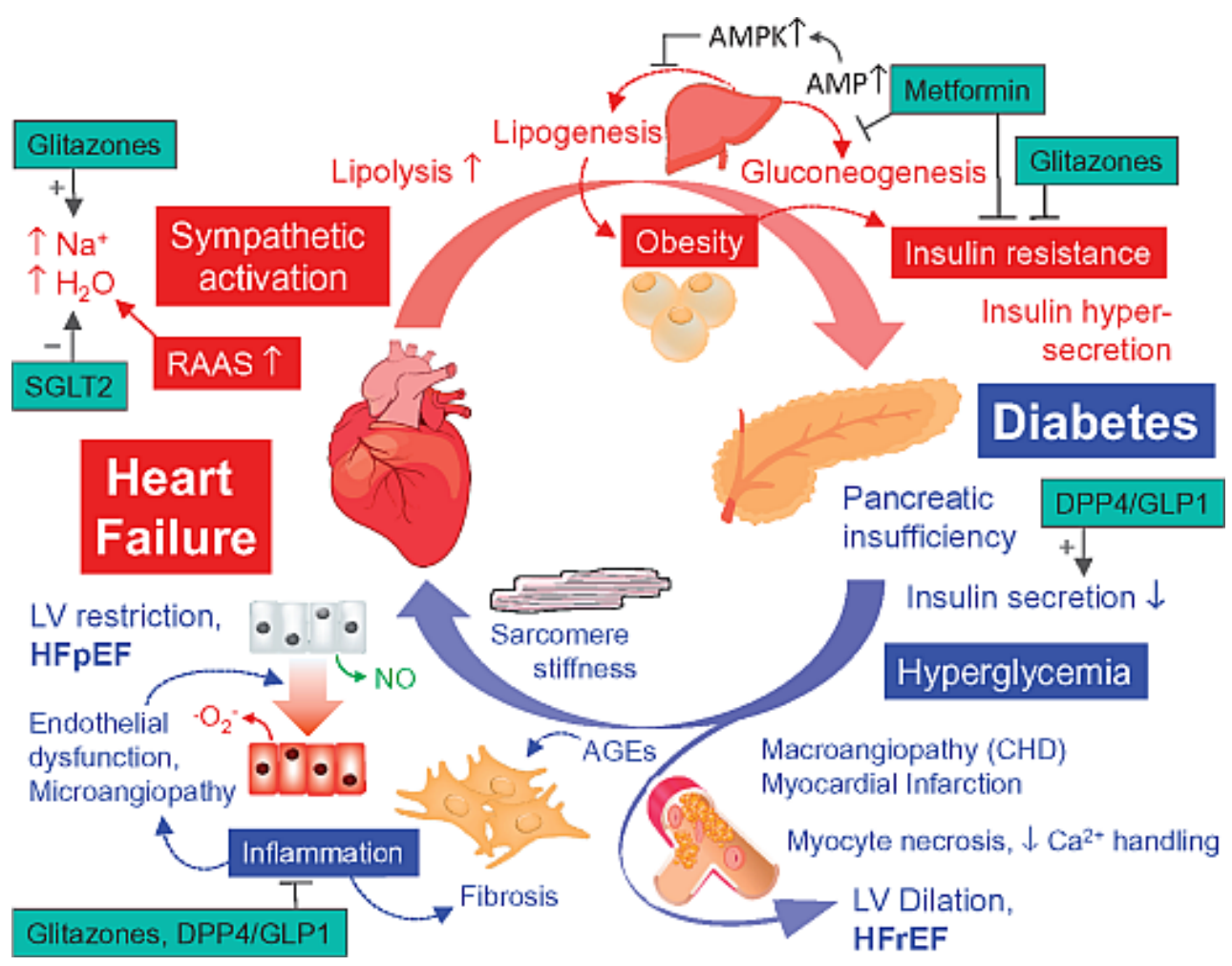

Fig.2.

A reduced stroke volume may occur as a result of a failure of systole, diastole or both. Increased end systolic volume is usually caused by reduced contractility. Decreased end diastolic volume results from impaired ventricular filling; this occurs when the compliance of the ventricle falls (i.e. when the walls stiffen). As the heart works harder to meet normal metabolic demands, the amount cardiac output can increase in times of increased oxygen demand (e.g., exercise) is reduced. This contributes to the exercise intolerance commonly seen in heart failure. This translates to the loss of one's cardiac reserve, or the ability of the heart to work harder during strenuous physical activity. Since the heart has to work harder to meet the normal metabolic demands, it is incapable of meeting the metabolic demands of the body during exercise.

A common finding in those with heart failure is an increased heart rate, stimulated by increased sympathetic activity $^{[2]}$ in order to maintain an adequate cardiac output. Initially, this helps compensate for heart failure by maintaining blood pressure and perfusion, but places further strain on the myocardium, increasing coronary perfusion requirements, which can lead to worsening of ischemic heart disease. Sympathetic activity may also cause potentially fatal abnormal heart rhythms. An increase in the physical size of the heart's muscular layer may occur. This is caused by the terminally differentiated heart muscle fibers increasing in size in an attempt to improve contractility. This may contribute to the increased stiffness and thus decrease the ability to relax during diastole. Enlargement of the ventricles can also occur and contributes to the enlargement and spherical shape of the failing heart. The increase in ventricular volume also causes a reduction in stroke volume due to mechanical and inefficient contraction of the heart ${ }^{[3]}$.

The general effect is one of reduced cardiac output and increased strain on the heart. This increases the risk of cardiac arrest (specifically due to abnormal ventricular heart rhythms) and reduces blood supply to the rest of the 
body. In chronic disease the reduced cardiac output causes a number of changes in the rest of the body, some of which are physiological compensations, some of which are part of the disease process:

- Arterial blood pressure falls. This destimulates baroreceptors in the carotid sinus and aortic arch which link to the nucleus tractus solitarii. This center in the brain increases sympathetic activity, releasing catecholamines into the bloodstream. Binding to alpha-1 receptors results in systemic arterial vasoconstriction. This helps restore blood pressure but also increases the total peripheral resistance, increasing the workload of the heart. Binding to beta-1 receptors in the myocardium increases the heart rate and makes contractions more forceful in an attempt to increase cardiac output. This also, however, increases the amount of work the heart has to perform.

- Increased sympathetic stimulation also causes the posterior pituitary to secrete vasopressin (also known as antidiuretic hormone or ADH), which causes fluid retention at the kidneys. This increases the blood volume and blood pressure.

- Heart failure also limits the kidneys' ability to dispose of sodium and water, which further increases edema $^{[4]}$. Reduced blood flow to the kidneys stimulates the release of renin - an enzyme which catalyses the production of the potent vasopressor angiotensin. Angiotensin and its metabolites cause further vasoconstriction, and stimulate increased secretion of the steroid aldosterone from the adrenal glands. This promotes salt and fluid retention at the kidneys.

- The chronically high levels of circulating neuroendocrine hormones such as catecholamines, renin, angiotensin, and aldosterone affect the myocardium directly, causing structural remodelling of the heart over the long term. Many of these remodelling effects seem to be mediated by transforming growth factor beta (TGF-beta), which is a common downstream target of the signal transduction cascade initiated by catecholamines ${ }^{[5]}$ and angiotensin $\mathrm{II},{ }^{[6]}$ and also by epidermal growth factor (EGF), which is a target of the signaling pathway activated by aldosterone ${ }^{[7]}$.

- Reduced perfusion of skeletal muscle causes atrophy of the muscle fibers. This can result in weakness, increased fatigability and decreased peak strength - all contributing to exercise intolerance ${ }^{[8]}$.

The increased peripheral resistance and greater blood volume place further strain on the heart and accelerates the process of damage to the myocardium. Vasoconstriction and fluid retention produce an increased hydrostatic pressure in the capillaries.

This shifts the balance of forces in favor of interstitial fluid formation as the increased pressure forces additional fluid out of the blood, into the tissue. This results in edema (fluid build-up) in the tissues. In right-sided heart failure, this commonly starts in the ankles where venous pressure is high due to the effects of gravity (although if the patient is bed-ridden, fluid accumulation may begin in the sacral region).

It may also occur in the abdominal cavity, where the fluid buildup is called ascites. In left-sided heart failure edema can occur in the lungs - this is called cardiogenic pulmonary edema. This reduces spare capacity for 
ventilation, causes stiffening of the lungs and reduces the efficiency of gas exchange by increasing the distance between the air and the blood.

The consequences of this are dyspnea (shortness of breath), orthopnea and paroxysmal nocturnal dyspnea.

The symptoms of heart failure are largely determined by which side of the heart fails. The left side pumps blood into the systemic circulation, whilst the right side pumps blood into the pulmonary circulation. Whilst left-sided heart failure will reduce cardiac output to the systemic circulation, the initial symptoms often manifest due to effects on the pulmonary circulation. In systolic dysfunction, the ejection fraction is decreased, leaving an abnormally elevated volume of blood in the left ventricle. In diastolic dysfunction, the end-diastolic ventricular pressure will be high. This increase in volume or pressure backs up to the left atrium and then to the pulmonary veins. Increased volume or pressure in the pulmonary veins impairs the normal drainage of the alveoli and favors the flow of fluid from the capillaries to the lung parenchyma, causing pulmonary edema. This impairs gas exchange. Thus, left-sided heart failure often presents with respiratory symptoms: shortness of breath, orthopnea, and paroxysmal nocturnal dyspnea.

In severe cardiomyopathy, the effects of decreased cardiac output and poor perfusion become more apparent, and patients will manifest with cold and clammy extremities, cyanosis, claudication, generalized weakness, dizziness, and fainting.

The resultant low blood oxygen caused by pulmonary edema causes vasoconstriction in the pulmonary circulation, which results in pulmonary hypertension. Since the right ventricle generates far lower pressures than the left ventricle (approximately $20 \mathrm{mmHg}$ versus around $120 \mathrm{mmHg}$, respectively, in the healthy individual) but nonetheless generates cardiac output exactly equal to the left ventricle, this means that a small increase in pulmonary vascular resistance causes a large increase in amount of work the right ventricle must perform. However, the main mechanism by which left-sided heart failure causes right-sided heart failure is actually not well understood. Some theories invoke mechanisms that are mediated by neurohormonal activation ${ }^{[9]}$.

\section{Systolic Dysfunction}

Heart failure caused by systolic dysfunction is more readily recognized. It can be simplistically described as a failure of the pump function of the heart. It is characterized by a decreased ejection fraction (less than $45 \%$ ). The strength of ventricular contraction is attenuated and inadequate for creating an adequate stroke volume, resulting in inadequate cardiac output. In general, this is caused by dysfunction or destruction of cardiac myocytes or their molecular components. In congenital diseases such as Duchenne muscular dystrophy, the molecular structure of individual myocytes is affected. Myocytes and their components can be damaged by inflammation (such as in myocarditis) or by infiltration (such as in amyloidosis). Toxins and pharmacological agents (such as ethanol, cocaine, doxorubicin, and amphetamines) cause intracellular damage and oxidative stress. The most common mechanism of damage is ischemia causing infarction and scar formation. After myocardial infarction, dead myocytes are replaced by scar tissue, deleteriously affecting the function of the myocardium. On echocardiogram, this is manifest by abnormal wall motion (hypokinesia) or absent wall motion (akinesia). 
Because the ventricle is inadequately emptied, ventricular end-diastolic pressure and volumes increase. This is transmitted to the atrium. On the left side of the heart, the increased pressure is transmitted to the pulmonary vasculature, and the resultant hydrostatic pressure favors extravasation of fluid into the lung parenchyma, causing pulmonary edema. On the right side of the heart, the increased pressure is transmitted to the systemic venous circulation and systemic capillary beds, favoring extravasation of fluid into the tissues of target organs and extremities, resulting in dependent peripheral edema.

\section{Diastolic Dysfunction}

Heart failure caused by diastolic dysfunction is generally described as the backward failure of the ventricle to adequately relax and typically denotes a stiffer ventricular wall. The "stiffness" and contractility of the ventricular walls in diastole was first described by Pierre-Simon Laplace. This causes inadequate filling of the ventricle and therefore results in an inadequate stroke volume (SV). SV is a mathematical term amenable to manipulation of many variables. The failure of ventricular relaxation also results in elevated end-diastolic pressures, and the end result is identical to the case of systolic dysfunction (pulmonary edema in left heart failure, peripheral edema in right heart failure). Diastolic dysfunction can be caused by processes similar to those that cause systolic dysfunction, particularly causes that affect cardiac remodeling. Diastolic dysfunction may not manifest itself except in physiologic extremes if systolic function is preserved.

The patient may be completely asymptomatic at rest. However, they are exquisitely sensitive to increases in heart rate, and sudden bouts of tachycardia (which can be caused simply by physiological responses to exertion, fever, or dehydration, or by pathological tachyarrhythmias such as atrial fibrillation with rapid ventricular response) may result in flash pulmonary edema. Adequate rate control (usually with a pharmacological agent that slows down AV conduction such as a calcium channel blocker or a beta-blocker) is, therefore, of key importance to preventing acute decompensation. Left ventricular diastolic function can be determined through echocardiography by measurement of various parameters such as the E/A ratio (early-to-atrial left ventricular filling ratio), the E (early left ventricular filling) deceleration time, and the isovolumic relaxation time.

\section{Dietary Recommendations}

\subsection{Foods to Include}

- Fish and flaxseed contain beneficial omega-3 fatty acids that reduce inflammation and LDL cholesterol, prevent blood clotting, and reduce the risk of heart disease.

- Garlic helps reduce cholesterol, thin the blood, and has antioxidant properties.

- Margarines, salad dressings, and spreads that contain phytostanols (plant substances) help lower cholesterol.

- Nuts (almonds and walnuts) help lower cholesterol levels. Nuts contain fibre and nutrients such as vitamin E, alpha-linolenic acid, magnesium, potassium, and arginine, which are important for heart health. Although nuts are high in calories, some studies have found that increasing nut consumption by several hundred calories per day does not cause weight gain. 
- Olive oil is a monounsaturated fat that can help reduce blood clots and lower LDL cholesterol.

- Soluble fibre which is found in oats, flaxseed, beans, psyllium, and fruits helps lower cholesterol levels. Insoluble fibre which is found in whole grains, vegetables, and fruits does not lower cholesterol, but studies have shown that it helps protect against heart disease. Aim for a total of $35 \mathrm{~g}$ of fibre daily.

- Fruits and vegetables also contain vital antioxidants and studies have shown that those who consume antioxidant-rich diets have lower rates of heart disease.

- Soy products (tofu, soybeans, miso, and soy protein powder) can help lower cholesterol and triglycerides.

- Yogurt and fermented milk products have been shown to lower cholesterol levels.

\subsection{Foods to avoid}

- Foods high in cholesterol should be minimized (organ meats, egg yolks, and whole milk products).

- Foods high in sugar and refined starches (white flour) can raise triglyceride and insulin levels, which increases the risk of heart disease.

- Salt causes water retention and increases the pressure inside your arteries. Reducing salt intake reduces blood pressure in most people.

- Avoid adding salt to foods and minimize eating processed and fast foods such as deli meats, snacks (chips, pretzels), french fries, and burgers.

- Saturated fat, which is present in animal foods (beef, pork, and dairy) and certain oils (palm oil), can raise cholesterol levels and is associated with increased heart disease risk.

\section{Lifestyle Suggestions}

- Don't smoke, and avoid second-hand smoke, as this increases many risk factors for heart disease.

- Lose excess weight, losing even 5 to 10 percent of excess weight can lower cholesterol and blood pressure.

- Exercise regularly, Moderate-intensity activities, such as brisk walking, biking, or swimming, can reduce cholesterol and blood pressure and help with weight management.

- Reduce stress levels, Stress causes the liver to increase the production of cholesterol, which is used to make stress hormones. Try meditation, exercise, or yoga to promote calming and relaxation.

- Manage your blood sugar levels, if you have diabetes or are at risk for diabetes, work on improving your blood sugar levels with exercise and a low-glycemic diet.

\subsection{Smoking and Heart Disease}

Smoking is a significant risk factor for heart disease as well as cancer. Smoking lowers the good HDL cholesterol, increases blood pressure, and causes serious damage to blood vessels. It is never too late to quit. Within one year of quitting, your risk of heart disease is half of that of a smoker's.

\section{OMEGADAY Softgels: The Superfood for Healthy Heart}

OMEGADAY softgels is a synergistic blend of Natural Astaxanthin, COQTEN Complex, Vitamin D3 \& Omega3 fatty acids. 


\section{Omega-3 -Fatty acids in OMEGADAY Softgels}

- Helps to provide cardiovascular health.

- Helps to lower total cholesterol, LDL Cholesterol \& triglycerides.

- Helps to reduce inflammation throughout the body.

- Helps to prevent skin disorders \& premature aging.

- Helps to protect the skin from damaging effects of UV Rays, Pollution, Stress, and Smoke.

Natural Astaxanthin in OMEGADAY Softgels

- Helps in neurovascular protection.

- Helps to provide skin defence by preventing UV induced and wrinkle formation and skin sagging.

- Helps to provide liver health and decreases inflammation.

- Helps to act as an immune system booster.

- Helps to provide cardiovascular health.

- Helps to prevent eye fatigue.

- Helps to improve fertility.

\section{COQTEN Complex in OMEGADAY Softgels}

- It helps to prevent the risk of heart disease.

- Fights gingivitis \& bleeding gums.

- Decreases the risk of infection.

- Improves brain performance \& memory.

\section{Vitamin D3 in OMEGADAY Softgels}

- Vitamin D3/Cholecalciferol present in omega day helps body to absorb calcium \& phosphorus.

- In the building and keeping of strong bones.

- In the prevention of bone loss (osteoporosis).

- In the prevention of diabetes mellitus 2 as it has an effect on insulin action.

\section{Composition}

\begin{tabular}{|c|c|c|}
\hline \multicolumn{3}{|c|}{ Supplement Facts } \\
\hline Serving Size : 1 Softgel & \multicolumn{2}{|c|}{ Serving per pack : 60} \\
\hline Each film coated tablet contains (approx.) & \multicolumn{2}{|c|}{$\%$ ICMR RDA* } \\
\hline $\begin{array}{l}\text { OMEGA } 3 \text { Fatty acids (Fish 0in) } \\
\text { (DHA-162 mE EPA-108 mgs) }\end{array}$ & $900 \mathrm{mg}$ & $* *$ \\
\hline Ubiquinone (COQTEN Complex) & $10,000 \mathrm{mcg}$ & ** \\
\hline Natural Astaxanthin (Heamatococcus Pluvialis) & 200 mcg & ** \\
\hline Vitamin D3 (as Cholecalciferol) & $400 \mathrm{IV}$ & 100 \\
\hline
\end{tabular}




\subsection{Pharmacological Action of Each Ingredient}

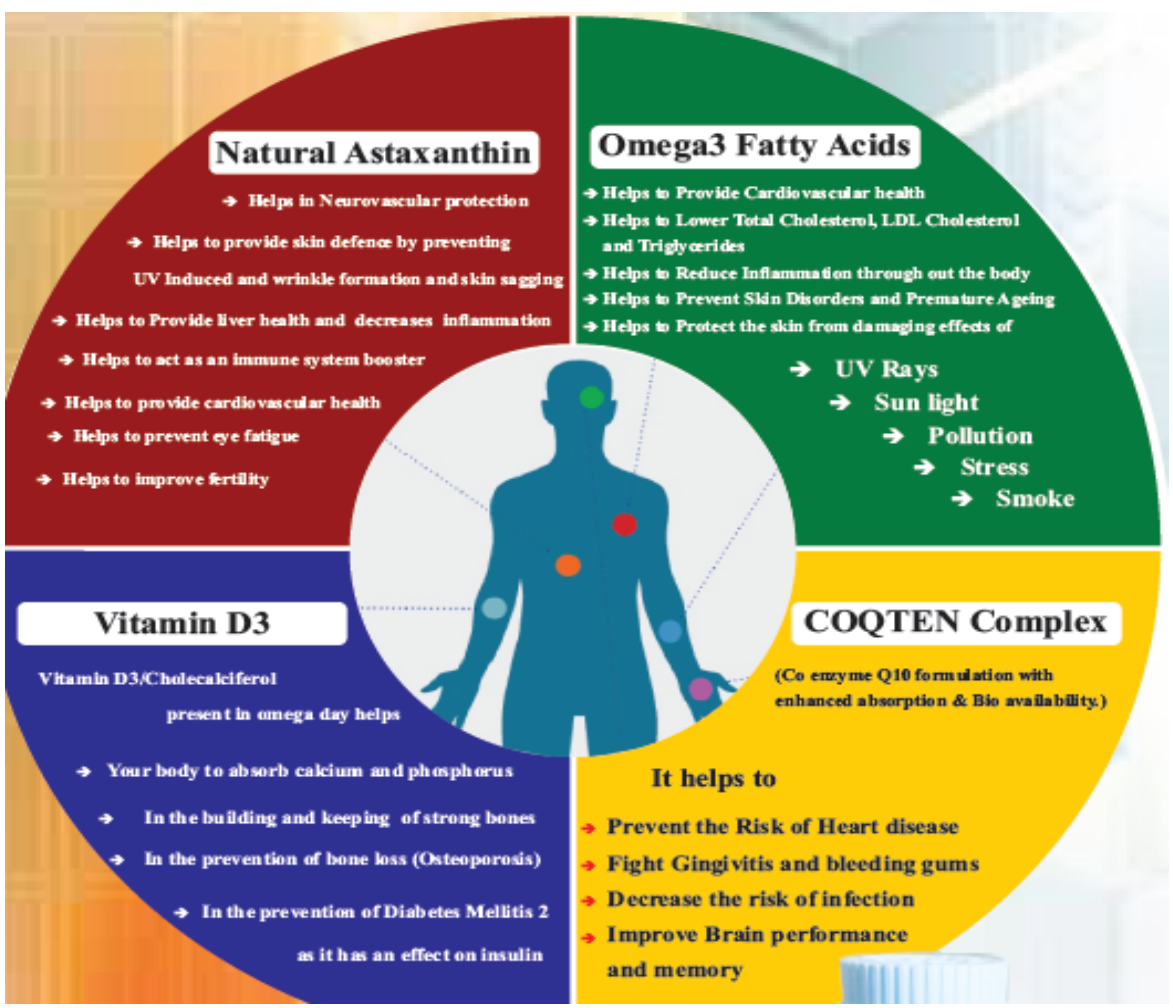

\subsection{Supplement Facts}

Presentation: 60 Softgels.

Usage: As a food supplement combination of antioxidants to improve health and vitality.

Contra-indications: Product is contra-indicated in persons with Known hypersensitivity to any component of the product hypersensitivity to any component of the product.

Recommended usage: Adults: two Softgels per day along with food.

"Do not exceed the recommended daily dose"

Administration: Taken by oral route at any time with food.

Precautions: Food Supplements must not be used as a substitute for a varied and balanced diet and a healthy lifestyle. This Product is not intended to diagnose, treat, cure or prevent any diseases. Do not exceed the recommended daily dose.

Warnings: If you are taking any prescribed medication or has any medical conditions or have any medical conditions (seizures) under age group 17 year always consults doctor or healthcare practitioner before taking supplements.

Side Effects: Mild side effects like nausea, headache and vomiting in some individuals have been reported.

Storage: Store in a cool, dry and dark place.

Keep out of reach of children. 
Declarations

\section{Source of Funding}

This research did not receive any specific grant from funding agencies in the public, commercial, or not-for-profit sectors.

\section{Competing Interests Statement}

The authors declare no competing financial, professional and personal interests.

\section{Ethical Approval}

Based on organizational guidelines.

\section{Consent for publication}

Authors declare that they consented for the publication of this research work.

\section{Availability of data and material}

Authors are willing to share data and material according to the relevant needs.

\section{References}

[1] Boron, Walter F., Boulpaep, Emile L., Medical Physiology: A Cellular and Molecular Approach (Updated ed.), Saunders, pp.533, 2005.

[2] Rang H.P., Pharmacology, Edinburgh: Churchill Livingstone, pp.127, 2003.

[3] Cardiac Pathophysiology in heart failure, GPnotebook.

[4] Tamparo, Carol, $5^{\text {th }}$ Ed.: Diseases of the Human Body, Philadelphia, PA: F.A. Davis Company, pp.329, 2011.

[5] Shigeyama J., Yasumura Y., Sakamoto A., Increased gene expression of collagen Types I and III is inhibited by beta-receptor blockade in patients with dilated cardiomyopathy, Eur. Heart J. 26 (24): 2698-705, 2005.

[6] Tsutsui H., Matsushima S., Kinugawa S., Angiotensin II type 1 receptor blocker attenuates myocardial remodeling and preserves diastolic function in diabetic heart, Hypertens. Res., 30 (5): 439-49, 2007.

[7] Krug A.W., Grossmann C., Schuster C., Aldosterone stimulates epidermal growth factor receptor expression, J. Biol. Chem., 278 (44): 43060-66, 2003.

[8] Systemic Pathophysiology in heart failure, GPnotebook.

[9] Hunter J.G., Boon N.A., Davidson S., Colledge N.R., Walker B., Davidson's principles \& practice of medicine, Elsevier/Churchill Livingstone, pp.544, ISBN 978-0-443-10057-4, 2006. 